\title{
A SARRUS-BASED PASSIVE MECHANISM \\ FOR ROTORCRAFT PERCHING
}

by

Michelle L. Burroughs

\begin{abstract}
A thesis submitted to the faculty of
The University of Utah

in partial fulfillment of the requirements for the degree of
\end{abstract}

Master of Science

Department of Mechanical Engineering

The University of Utah

December 2014 
Copyright @ Michelle L. Burroughs 2014 All Rights Reserved 


\section{The University of Utah Graduate School}

\section{STATEMENT OF THESIS APPROVAL}

The thesis of

Michelle L. Burroughs

has been approved by the following supervisory committee members:

Jake J. Abbott

Mark A. Minor

Sanford G. Meek
, Chair

, Member

, Member

Tim Ameel

, Chair/Dean of $9 / 1 / 14$

Date Approved 9/1/14

Date Approved $9 / 1 / 14$ Date Approved

$9 / 1 / 14$
Date Approved

and by

the Department/College/School of Mechanical Engineering

and by David B. Kieda, Dean of The Graduate School. 


\begin{abstract}
Flying rotorcraft, such as helicopters and quadrotors, can gather useful information without the need for human presence, but they consume a great deal of power and have limited on-board energy resources. Our work aims to provide a passive perching mechanism so that a rotorcraft is able to grip branch-like perches and resist external wind disturbances, using only the weight of the rotorcraft to maintain the grip. Deviating from previous bio-inspired approaches, in this thesis, we propose a mechanism that incorporates a Sarrus linkage to convert the weight of the rotorcraft into grip force. We provide an analysis of the mechanism's kinematics, we present the static force equations that describe how the weight of the rotorcraft is converted into grip force onto a cylindrical perch, and we describe how grip forces relate to the ability to reject horizontal disturbances such as wind gusts. The mechanism is then optimized for use on a single perch size, and then for a range of perch sizes. We conclude by constructing a prototype mechanism, and we demonstrate its use with a remote-controlled helicopter.
\end{abstract}




\section{CONTENTS}

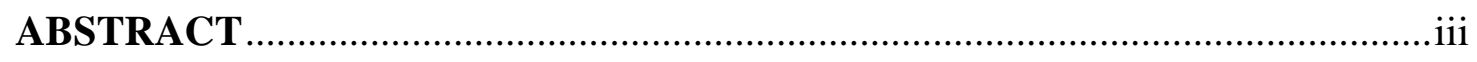

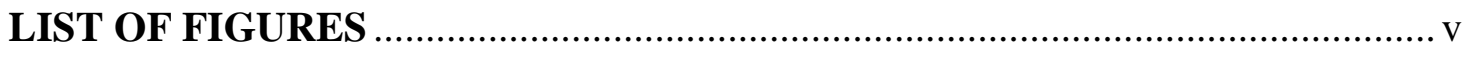

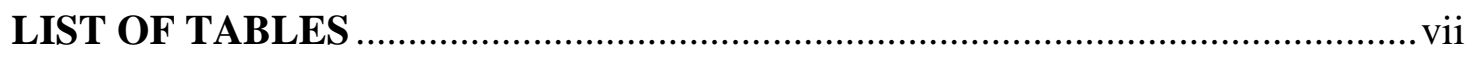

ACKNOWLEDGEMENTS …...........................................................................ii

\section{CHAPTERS}

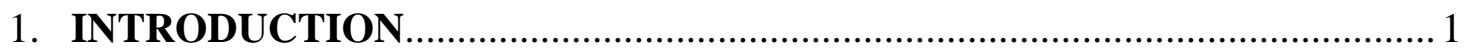

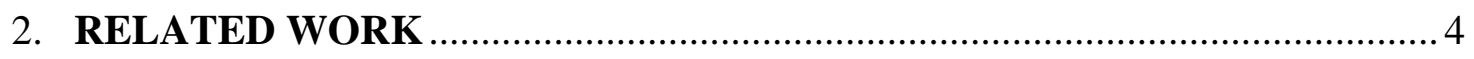

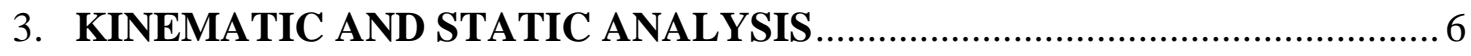

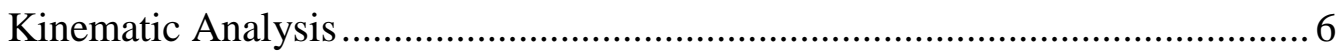

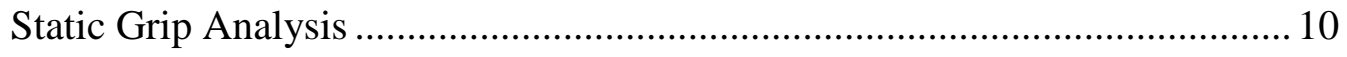

Disturbance Rejection .................................................................................... 12

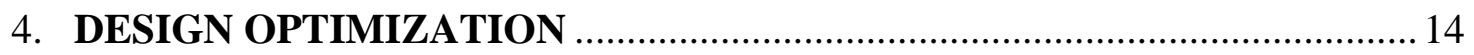

Optimization for a Single Perch Size................................................................ 14

Optimizing for a Range of Perch Sizes............................................................. 22

5. PROTOTYPE AND EXPERIMENTAL VERIFICATION ……....................29

6. CONCLUSION AND FUTURE WORK ……………….................................. 38

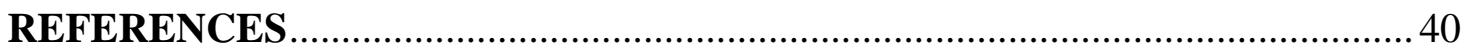




\section{LIST OF FIGURES}

1. Prototype mechanism attached to helicopter............................. 3

2. Sarrus mechanism............................................... 3

3. Kinematic description of MH6arrus-based mechanism FRQMGHHGin this thesis, Z ILXMHrotorcraft's weight $W$ causing the mechanism to grip on a cylindrical

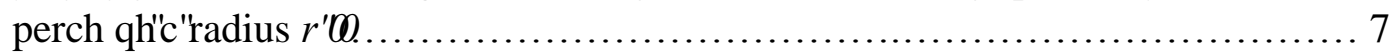

4. Force and moment balance of 6arrus-based mechanism................... 9

5. Comparison of initial nominal design (a) and optimal design (b) for a single

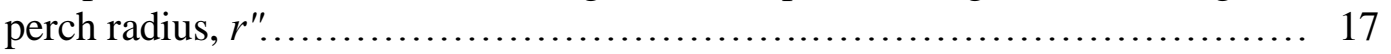

6. Varying design parameters in nominal design for a single perch size......... 19

7. Varying design parameters in optimal design for a single perch size......... 20

8. Normalized force disturbance $\tilde{F}_{D}$ vs. distance to the center of pressure $\tilde{a} \ldots \ldots 21$

9. Minimum relative perch size $s$ vs. minimum grip angle $\theta_{\min } \ldots \ldots \ldots \ldots \ldots \ldots$

10. Normalized Iorce Gsturbance vs. Lelative Serch Vze for Iour Gfferent P echanisms that Kave Hach Eeen Rptimized for a Wecified Lange of Serch vizes................................................................. 25

11. Normalized force disturbance vs. normalized link length for four different mechanisms that have each been optimized for a specified range of perch sizes............................................................... 26

12. Normalized force disturbance vs. minimum grip angle for four different mechanisms that have each been optimized for a specified range of perch sizes.................................................................

13. Helicopter in wind tunnel......................................... 30

14. Drag force vs. wind speed obtained in a wind tunnel.................... 30 
15. Lift Iorce vs. Z ind \peed obtained in a wind tunnel .................... 31

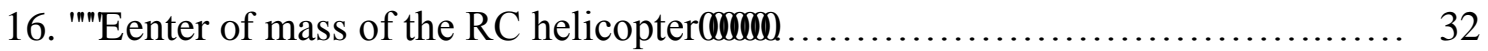

17. Model of the prototype, optimized for the range of perch sizes of $s=\left[\begin{array}{ll}0.51] \ldots 34 \\ \text { 1 }\end{array}\right.$

18. Video images of the descent and ascent of helicopter with attached perching

P FFWDUP [on $42 \mathrm{~mm}$ and $21 \mathrm{~mm}$ diameter PVC perches.................. 


\section{LIST OF TABLES}

1. PDUP HMUXYGIIRUSrototype design .............................. 34 


\section{ACKNOWLEDGEMENTS}

I would like to thank Dr. Jake J. Abbott and Dr. Mark M. Minor for their assistance and mentorship throughout the research presented in this thesis. I would also like to thank Beau Freckleton for his development of a solid model of the mechanism and continuous participation with the project, Tom Slowick for his assistance fabricating our prototypes, and Kevin Marett for his assistance collecting wind-tunnel data. 


\section{CHAPTER 1}

\section{INTRODUCTION}

Flying robots, including rotorcraft and fixed-wing aircraft, have an increasing prevalence in a variety of applications because of their ability to gather useful information without the need for human presence. However, flying robots consume a great deal of power, yet have limited on-board energy resources. This makes hovering an inefficient method for data gathering. Perching on a structure (e.g., a tree branch, the roof of a building) allows a rotorcraft to gather information without consuming power, and even potentially recharge the energy source.

Our work aims to provide a passive perching mechanism so that a rotorcraft, such as a helicopter or quadrotor, is able to grip branch-like perches and resist external wind disturbances, using only the weight of the rotorcraft to maintain the grip. In our previous designs, we explored mechanisms inspired by the method used by songbirds to sleep while perching [0][2]. Songbirds have a tendon on the rear side of the ankle that allows them to automatically grip as the bird relaxes, with the weight of the bird causing the legs to bend, putting tension on the tendon, and causing the toes to grip. When the bird wants to take off, muscles are used to straighten the legs, releasing the grip.

In this thesis, we propose a new mechanism that is neither avian-inspired nor tendon-based, but is designed to accomplish the same passive perching as our previous designs (Fig. 1). The new mechanism incorporates a Sarrus linkage to convert the weight 
of the rotorcraft into grip force. This one-degree-of-freedom mechanical linkage enforces linear translational motion between two parallel plates (Fig. 2).

We use the linkage to convert the translational motion of the descent of the rotorcraft during a perching maneuver into angular motion of the connecting links with rigidly attached toes, resulting in a grip. Similarly, the translational ascent of the rotorcraft naturally releases the grip. The Sarrus linkage exhibits a high mechanical advantage in its collapsed state, such that the downward force exerted by the rotorcraft's weight is amplified in the toes' grip.

In this thesis, we begin with an analysis of the kinematics of our mechanism. We then present the static force equations that describe how the weight of the rotorcraft is converted into grip force onto a perch, and we describe how grip forces relate to the mechanism's ability to reject horizontal disturbances such as wind gusts. The mechanism is then optimized for use on a single given perch size. The optimization is then expanded to consider a range of perch sizes. We conclude by constructing a prototype mechanism, and we demonstrate its use with a remote-controlled helicopter. 


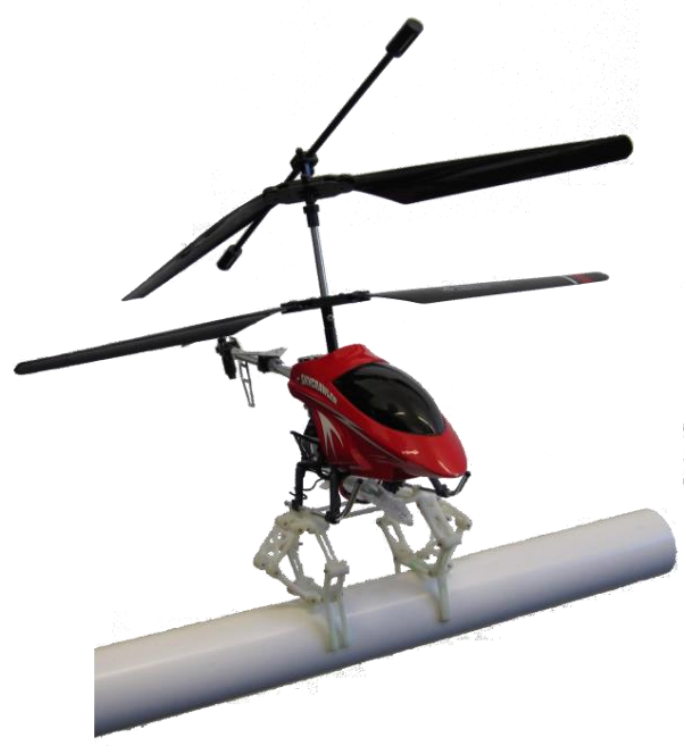

Fig. 1. Prototype mechanism attached to helicopter. Our mechanism, shown here attached to the skids on the bottom of an RC helicopter, uses a bilateral configuration of Sarrusbased linkages to passively grip cylindrical perches. The mechanical advantage converts the weight of the helicopter into an amplified force exerted onto the perch grip, which allows the system to resist disturbances.

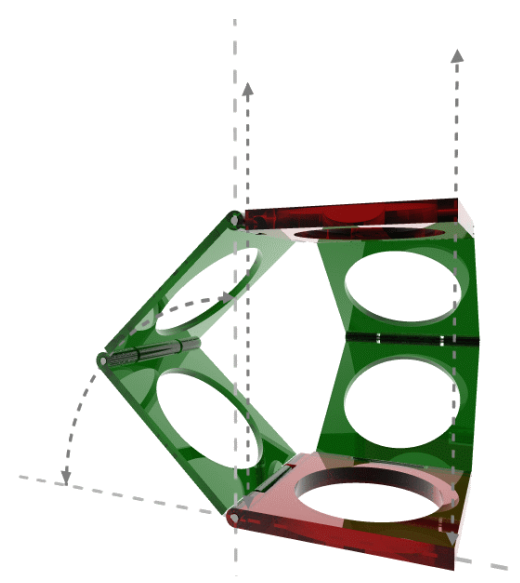

Fig. 2. Sarrus mechanism. A Sarrus linkage converts the pure translational motion between the top and bottom plates (red) into angular motion of the plates in the connecting linkages (green). The two connecting linkages must be out-of-plane to keep the top and bottom plates parallel. The mapping between the angular motion of the connecting plates and the translational motion between the top and bottom plates is nonlinear. Public domain image [3] http://en.wikipedia.org/wiki/Sarrus_linkage. 


\section{CHAPTER 2}

\section{RELATED WORK}

Many prior works have considered perching for either fixed-wing aircraft or rotorcraft, approached through various combinations of mechanism design, sensing, and control algorithms. Perching has been explored for a variety of surface orientations ranging from horizontal to vertical, and for a variety of surface geometries ranging from flat surfaces to cylindrical structures such as branches and power lines.

Some research on perching of fixed-wing aircraft has been largely based on sensing and control of the aircraft itself during a perching maneuver. Larson [16] developed a fixed-wing aircraft with an ultrasonic distance sensor capable of perching on both vertical and horizontal surfaces if a gripper were attached. Moore and Tedrake [6][7] developed a technique for perching at an incline on power lines using landing gear and magnetic localization. Hurst et al. [25], Hurst and Garcia [5], Gomez and Garcia [26], and Robertson and Reich [10] developed control schemes for morphing aircraft that use bird-inspired wings and tails to perch on flat, horizontal surfaces.

In other research, perching of fixed-wing aircraft has been approached through the design of passive and active perching mechanisms. Several groups have used passive perching mechanisms attached to fixed-wing aircraft such as microspines, hooks, and

adhesives. Desbians et al. [8][9][12] and Glassman et al. [13] developed fixed-wing aircraft with microspines attached to the bottom of the fuselage to adhere to vertical 
surfaces. Similarly, Anderson et al. [14] utilized a sticky pad attached to the bottom of the fuselage to adhere to vertical surfaces. Cory and Tedrake [12] developed a fixed-wing robot with a latching hook that is used to perch on a horizontal string. Other groups have developed active mechanisms. Nagendran et al. [27] developed bio-inspired legs to grab and perch on flat, horizontal surfaces. Bachmann et al. [17] developed a crawling, fixedwing robot intended to land on a large, horizontal surface area.

Researchers have also equipped rotorcraft with active perching mechanisms. Kovac et al. [18] and Mellinger et al. [22] developed perching mechanisms that use small barbs that enable perching on flat surfaces at a variety of inclines. In other work, researchers have developed active mechanisms to be used for grasping, but which could also be used for perching. Pounds et al. [23] and Ghodiak et al. [24] developed underactuated compliant hand-like graspers that enable a rotorcraft to grasp and carry an object during flight. Thomas et al. [21] recently equipped a quadrotor with an actuated arm capable of grasping objects at high horizontal speed, using an approach inspired by raptors.

A few groups have considered passive rotorcraft perching in a similar spirit to the work reported in this thesis. Danko et al. [4] and Goldin [11] developed rotorcrafts with onboard sensors for autonomous perching on flat, horizontal surfaces, but with no dedicated perching mechanism. Daler et al. [15] developed a mechanism comprising fibre-based adhesive that enables perching on flat, vertical surfaces. In work that has a similar motivation to ours, Culler et al. [20] developed a gripping mechanism for a quadrotor, which uses a compliant snapping claw mechanism that is triggered upon landing on a branch-like structure. 


\section{CHAPTER 3}

\section{KINEMATIC AND STATIC ANALYSIS}

In this section, we first describe the design of our proposed mechanism, and report on its governing kinematic equations. Next, we report on the static equations that describe how the weight of the rotorcraft is converted into grip force between the mechanism and the perch. Finally, we describe how the resulting grip force relates to the maximum applied horizontal disturbance force on the rotorcraft that can be successfully rejected, such as that due to wind.

\section{$\underline{\text { Kinematic Analysis }}$}

Our Sarrus-based perching mechanism, shown in Fig. 3, is described through a set of constant geometric parameters, which include the base length $b$, the link length $L$, the rigid toe angle $\Psi$, and the toe length $T$. For the configuration of the mechanism described in this thesis, the mechanism is symmetric, with the top and bottom plates assumed to be of equal length, and all of the top and bottom side links assumed to be of equal length; these assumptions could be relaxed in future work. The top and bottom plates always remain parallel throughout the perching maneuver, which is enforced by an out-of-plane linkage that is identical to the two side linkages shown in Fig. 3 but without the toe (as in Fig. 2).

Additional parameters describe the configuration of the mechanism when 


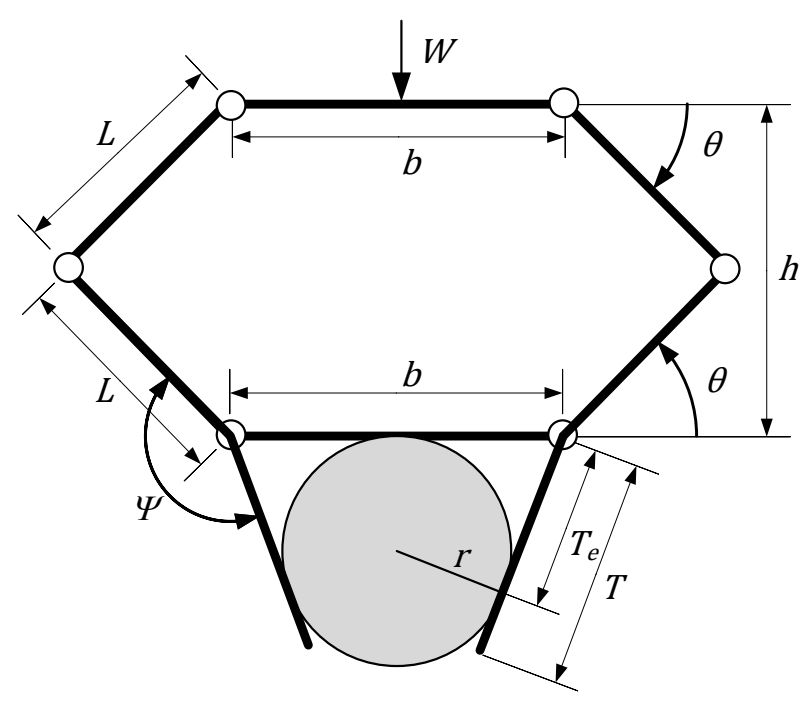

Fig. 3. Kinematic description of the Sarrus-based perching mechanism considered in this thesis, with the rotorcraft's weight $W$ causing the mechanism to grip on a cylindrical perch of a radius $r$. The mechanism is described by a set of constant geometric parameters: the base length $b$, the link length $L$, the toe length $T$, and the rigid toe angle $\Psi$ between the toe and the bottom-side link. The mechanism is symmetric, and an out-ofplane linkage that is identical to the two side linkages (not shown) enforces that the top and bottom plates remain parallel as in Fig. 2. An additional set of parameters are used to describe the configuration of the mechanism on a given perch: the grip angle $\theta$, the height $h$, and the effective toe length $T_{e}$. Noncompliant and frictionless pin joints are assumed between all rigid links, indicated with small circles. Note that a bottom-side link and a toe form a single rigid link, which is joined to the bottom plate with a pin joint.

collapsed on a perch. The effective toe length $T_{e}$ describes the length of the toe between the bottom joint and the toe's contact point with the perch. The grip angle $\theta$ describes the angle between the bottom side link and the horizontal. $\theta=0$ and $\theta=\pi / 2$ rad both correspond to toggle positions in the mechanism that should be avoided. The height $h$ of the mechanism defines the distance between the top and bottom plates. The grip angle is related to the height through the link length $L$ using the law of cosines:

$$
h=2 L \sin (\theta) \Leftrightarrow \theta=\sin ^{-1}\left(\frac{h}{2 L}\right)
$$


Next, to find the location where the toe contacts the perch, we define a new angle $\beta$ that we call the toe contact angle (Fig. 4). To solve for $\beta$, we can analyze symmetric halves of the area between a toe and the bottom plate through an angle $\alpha$ (Fig. 4). Geometrically, the angle $\alpha$ is a function of the base length $b$ and the perch radius $r$ :

$$
\alpha=\tan ^{-1}\left(\frac{2 r}{b}\right)
$$

To solve for the toe contact angle, $\beta$, we relate the Cartesian coordinates of the toe contact point to the angle $\alpha$, base length $b$, and perch radius $r$ :

$$
\begin{aligned}
& x_{\beta}=\frac{b}{2} \cos (2 \alpha) \\
& y_{\beta}=\frac{b}{2} \sin (2 \alpha) \\
& \beta=\tan ^{-1}\left(\frac{x_{\beta}}{y_{\beta}}\right)
\end{aligned}
$$

After substitution and simplification, we arrive at an equation for $\beta$ based on known parameters:

$$
\beta=\frac{\pi}{2}-2 \tan ^{-1}\left(\frac{2 r}{b}\right)
$$

We can see that the equation for the toe contact angle is only a function of one dimensionless variable: the ratio of the perch diameter to the base length. The solution becomes degenerate when the perch diameter is equal to the base length; for this trivial case, $\beta=0$. The grip angle $\theta$ is related to the toe contact angle $\beta$ through the rigid toe angle $\Psi$ as,

$$
\theta=\Psi-\beta-\frac{\pi}{2}
$$

Because the toe's contact point and bottom plate's contact point are equidistant 


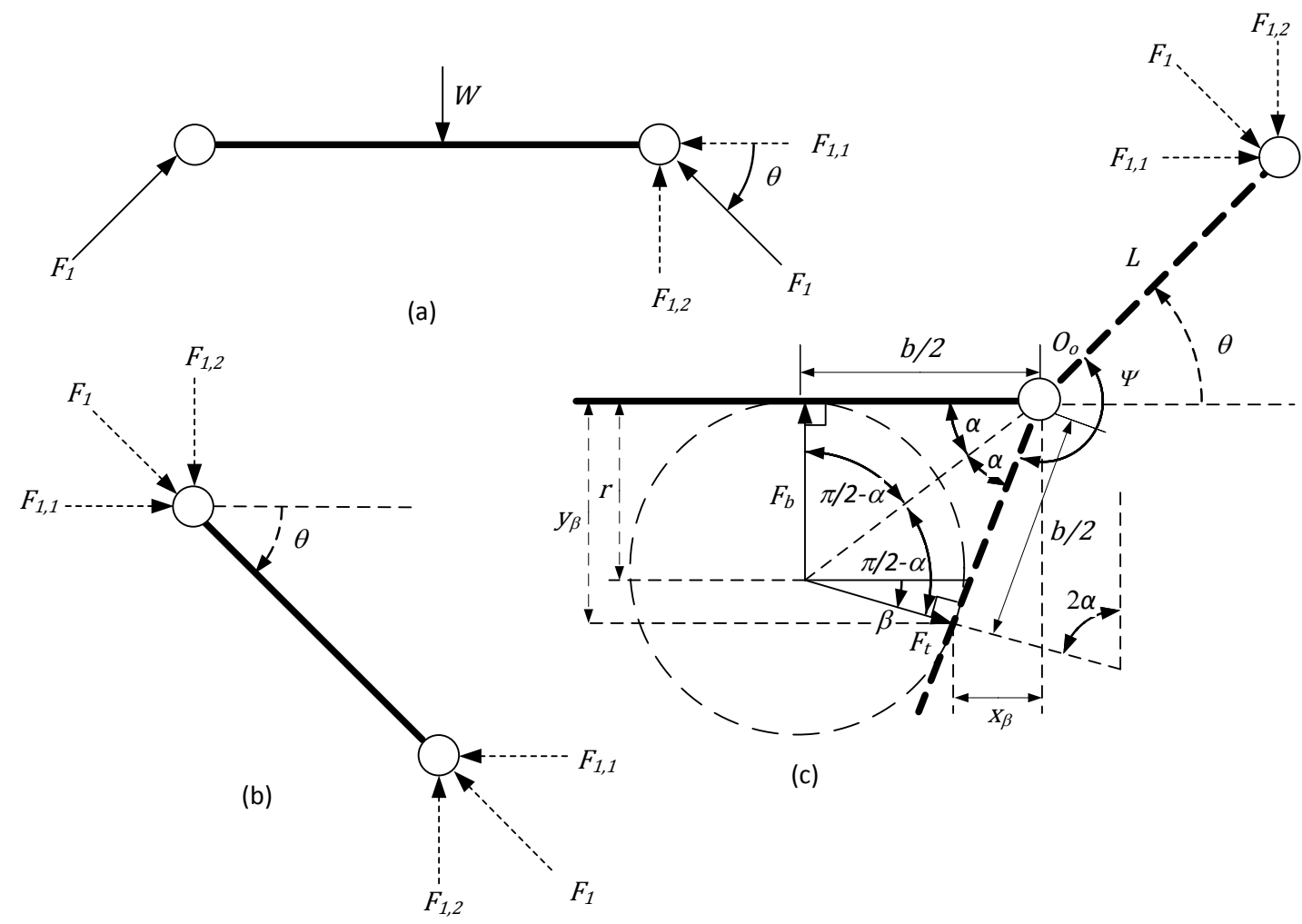

Fig. 4. Force and moment balance of Garrus-based mechanism. Right side of symmetrical Sarrus-based perching mechanism, broken into three components; (a) the top plate, (b) the top side linkage, and (c) the bottom half of the mechanism, which includes the bottom side linkage and its rigidly attached toe, attached to the bottom plate at a pin joint. Small circles represent pin joints.

from joint $O_{o}$, it is apparent that the effective toe length is $T_{e}=b / 2$ for any perch size. From this result, it is clear that the largest perch size that can be surrounded and gripped by the toes as shown in Fig. 3 is bounded by $r=b / 2$ (this result is intuitive for straight toes). 


\section{$\underline{\text { Static Grip Analysis }}$}

With an understanding of the kinematic configuration of the perching mechanism, we can now perform a static analysis that relates the weight of the rotorcraft to the grip force that the mechanism applies to the perch. We can deconstruct the mechanism into two symmetrical halves, and then work through the linkage on the right side to find the forces throughout the linkage (Fig. 4). We describe the force acting between the top plate and the top side link as $F_{1}$, which we decompose into its Cartesian force components $F_{1,1}$ and $F_{1,2}$ (all assumed to be positive when in compression). If we perform a static force and moment balance on the top side link (Fig. 4(b)), we find that the force $F_{1}$ must be directed along the link since the pin joints on the two ends cannot support a moment. Therefore, we know that $F_{1}$ is applied to the top plate at the grip angle $\theta$ (Fig. 4(a)). Using a static force balance on the top plate, we can solve for $F_{1}$ and its Cartesian components as a function of the rotorcraft's weight $W$ :

$$
\begin{gathered}
F_{1,2}=\frac{W}{2}=F_{1} \sin (\theta) \\
F_{1}=\frac{W}{2 \sin (\theta)} \\
F_{1,1}=\frac{F_{1,2}}{\tan (\theta)}=\frac{W}{2 \tan (\theta)} \\
F_{t}\left(\frac{b}{2}\right)-F_{1,1}(L \sin (\theta))-F_{1,2}(L \cos (\theta))=0
\end{gathered}
$$

Now that we have the internal linkage forces expressed in terms of the weight of the rotorcraft and the grip angle, we can use a moment balance about joint $O_{o}$ to the find the compressive force $F_{t}$ that the toe applies to the perch resulting in an equation for the force that a toe applies to the perch, in which the weight of the rotorcraft enters linearly: 


$$
F_{t}=W\left(\frac{2 L}{b} \cos (\theta)\right)
$$

with $\theta$ already defined as a function of known mechanism parameters in Eq. (7).

We have assumed until now that there is one toe on each side of the perch. In general, there could be several toes on a given side of the perch, in which case the force $F_{t}$ can be assumed to be distributed equally between the toes.

To find the compressive force $F_{b}$ that the bottom plate applies to the perch, we first perform a static force balance on the bottom plate and find that the vertical force $F_{b}$ must be shared equally between the two pin joints of the bottom plate due to symmetry. Next we perform a static force balance in the vertical direction on the bottom side link:

$$
\begin{gathered}
\frac{F_{b}}{2}-F_{1,2}-F_{t} \sin (\beta)=0 \\
F_{b}=W\left(1+2\left(\frac{2 L}{b}\right) \cos (\theta) \sin (\beta)\right)
\end{gathered}
$$

with $\beta$ and $\theta$ defined as functions of mechanism parameters in Eq. (6) and Eq. (7), respectively. Just as the term $2 r / b$ was found to be important for characterizing the toe contact angle $\beta$, we find that the parameter $2 L / b$ is important for characterizing the grip force of the mechanism, both from the toes and from the bottom plate.

We would like $F_{b}$ to remain positive (i.e., in compression) to ensure that the bottom plate maintains in contact with the perch, both so that it is contributing to the grip and so that our kinematic analysis remains valid. This requires:

$$
\cos (\theta)>-\frac{b}{4 L \sin (\beta)}
$$

We see that this condition is always satisfied when $\beta$ is constrained to be in the range $0<\beta<\pi / 2$ by design, assuming that $\theta$ falls within the normal operating range of the 
Sarrus linkage: $0<\theta<\pi / 2$. Enforcing this assumption requires that we constrain $\Psi$ for any given $\beta$ to be in the range:

$$
\beta+\frac{\pi}{2}<\Psi<\beta+\pi
$$

We will find in Chapter 4 that as our range of desired perch sizes (and thus, the range of expected $\beta$ ) increases, our range of acceptable $\Psi$ values decreases.

\section{Disturbance Rejection}

In this section, we describe how the grip forces between the mechanism and the perch relate to the ability of the mechanism to reject horizontal disturbance forces (e.g., wind) on the rotorcraft. We will assume that a disturbance force $F_{D}$ is applied horizontally at some distance $a$ above the top plate (e.g., at the center of pressure of the rotorcraft). This force creates a disturbance moment about the perch:

$$
M_{D}=F_{D}(a+h+r)
$$

that must be counteracted by the grip (this equation assumes negligible thickness of the top and bottom plates). We will assume a coefficient of static friction $\mu$ at the perchmechanism interface. The maximum static moment that can be rejected by the grip forces, which act at a moment arm $r$, is given by:

$$
M_{D, \max }=\mu r\left(F_{b}+2 F_{t}\right)
$$

which, after substitutions, becomes:

$$
M_{D, \max }=\mu W r\left(1+\left(\frac{4 L}{b}\right) \cos (\theta)(1+\sin (\beta))\right)
$$

Our mechanism will be able to reject any disturbance moment that is less than this maximum: $M_{D}<M_{D . \max }$. We can combine the above equations to solve for the upper 
bound on the horizontal disturbance force that can be rejected:

$$
F_{D, \max }=\frac{\mu W r\left(1+\left(\frac{4 L}{b}\right) \cos (\theta)(1+\sin (\beta))\right)}{a+2 L \sin (\theta)+r}
$$

with $\beta$ and $\theta$ defined as functions of mechanism parameters in Eq. (6) and Eq. (7), respectively. Note that the rotorcraft's weight $W$ and the coefficient of static friction $\mu$ both enter into the equation linearly. Equipped with an equation that describes the maximum disturbance force that can be rejected as a function of the parameters of our mechanism, we are able to optimize the design of our mechanism using robustness to disturbances as our design metric. 


\section{CHAPTER 4}

\section{DESIGN AND OPTIMIZATION}

In this section, we describe the process of defining the optimal perching mechanism, first for a single perch, and then for a specified range of perch sizes.

\section{Optimization for a Single Perch Size}

To optimize for a single perch size, we first define the range of parameters for which the mechanism/rotorcraft system will achieve a valid grasp (as depicted in Fig. 3). Then we examine each parameter individually, and find an optimal solution that maximizes the disturbance force that can be rejected.

Before performing the optimization of our mechanism, we first recognize that the rotorcraft's weight and the coefficient of friction between the toes and the perch both enter into the maximum disturbance force linearly, and we can therefore optimize a nondimensional maximum disturbance force:

$$
\tilde{F}_{D}=\frac{F_{D, \max }}{\mu W}
$$

Note we drop the "max" subscript in the nondimensional variable for brevity. We can also normalize our various length parameters by the perch radius, which can be seen by dividing both the numerator and denominator of Eq. (20) by r:

$$
\tilde{L}=\frac{L}{r}, \quad \tilde{b}=\frac{b}{r}, \quad \tilde{a}=\frac{a}{r}
$$


The result is a fully nondimensional equation for the maximum disturbance force that can be rejected:

$$
\tilde{F}_{D}=\frac{4 \tilde{L} \cos (\theta)(1+\sin (\beta))+\tilde{b}}{\tilde{a} \tilde{b}+2 \tilde{L} \tilde{b} \sin (\theta)+\tilde{b}}
$$

Our goal during optimization will be to maximize this quantity.

First we consider the effect of the normalized link length $\tilde{L}$. In the limit as $\tilde{L}$ becomes very small, the perching mechanism has no role in rejecting a disturbance force, and the rotorcraft is essentially balancing atop the perch:

$$
\lim _{\tilde{L} \rightarrow 0} \tilde{F}_{D}=\frac{1}{\tilde{a}+1}
$$

In the limit as $\tilde{L}$ becomes very large, there is an upper bound to the force that can be rejected:

$$
\lim _{\tilde{L} \rightarrow \infty} \tilde{F}_{D}=\frac{2(1+\sin (\beta))}{\tilde{b} \tan (\theta)}
$$

As we increase the link length, the disturbance force that can be rejected increases, but there is a diminishing return as we asymptotically approach the upper bound in (25). Since an increase in link length will have an accompanying increase in weight that must be lifted by the rotorcraft, we must be conscientious of this trade-off. In the general case where link length is neither extremely short nor extremely long, the relationship between the various design parameters and the maximum disturbance force is nontrivial.

We see that (at least for very long link lengths) there is clear push to minimize the grip angle $\theta$ to increase disturbance rejection, which is not confounded by other factors. This corresponds to the mechanism collapsing down into its toggle position. Because of the high mechanical advantage near the toggle position, the weight of the rotorcraft could 
potentially deform the links or joints and violate the fundamental kinematics of the mechanism. Therefore, we introduce a new design parameter: the minimum grip angle $\theta_{\min }$ (Fig. 5). This parameter establishes how close we will allow the mechanism to get to its fully collapsed toggle position (i.e., horizontal). We will use the same angle to establish how close we will allow the mechanism to get to its fully open toggle position (i.e., vertical) when the mechanism hangs below the rotorcraft; in future work, these values could be set independently.

We must now update our relationship for the allowable rigid toe angle from (16) to account for the user-defined minimum grip angle. We will also incorporate our solution for $\beta$ from (6), as well as our $\Psi$ normalization of (23). The resulting limit on $\Psi$ is:

$$
\pi-2 \tan ^{-1}\left(\frac{2}{\tilde{b}}\right)+\theta_{\min }<\Psi<\frac{3 \pi}{2}-2 \tan ^{-1}\left(\frac{2}{\tilde{b}}\right)-\theta_{\min }
$$

The size of the maximum range of acceptable rigid toe angles $\Psi$ is thus always $\pi / 2-$ $2 \theta_{\min }$ for any given perch size, with the values for the base length $\tilde{b}$ and minimum grip angle $\theta_{\text {min }}$ defining the boundaries of this range. As an example, consider a base length $\tilde{b}=2$ (in which the base length is equal to the diameter of the perch) and a minimum grip angle $\theta_{\min }=0$ : the range of acceptable toe angles is from $\pi / 2$ to $\pi \mathrm{rad}$ and the corresponding range for the toe contact angle $\beta$ is from 0 to $\pi / 2 \mathrm{rad}$. As the size of the desired perch decreases relative to the base length, but with the minimum grip angle maintained at $\theta_{\min }=0$, the lower bound on acceptable $\Psi$ evolves toward $\pi$ radians, with the size of the range remaining as $\pi / 2 \mathrm{rad}$.

To explore the effect of each of the remaining parameters, we begin by considering a nominal set of values that constitute an initial "reasonable" design, and then 


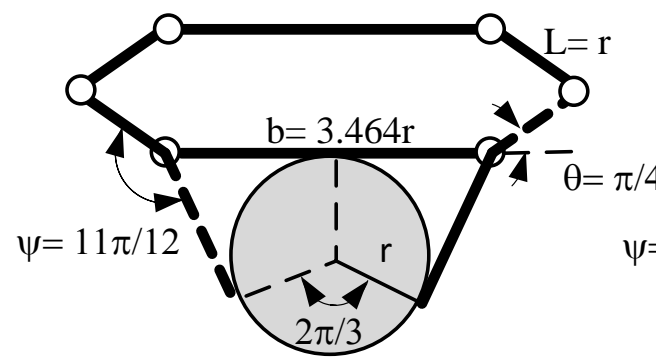

(a)

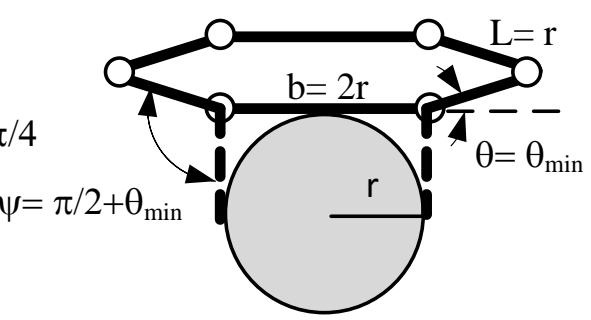

(b)

Fig. 5. Comparison of initial nominal design (a) and optimal design (b) for a single perch radius, $r$. The optimal configuration has minimized the optimization parameters base length $b$ and rigid toe angle $\psi$ to the minimum boundary limits to create the highest force disturbance output $F_{D}$. The link length $L$ and grip angle $\theta_{\min }$ are set arbitrarily.

vary each parameter individually. Our nominal design is based on the idea that equidistant points of contact around the perch would provide a good grasp while perching (Fig. 5(a)). Thus, the toe collision angle is set at $\beta=\pi / 6$ rad to create an equal distribution of contact points between the toes and the bottom plate (i.e., contact points surrounding the perch every $120^{\circ}$ ). We set the nominal minimum grip angle at $\theta_{\min }=$ $\pi / 18 \mathrm{rad}\left(10^{\circ}\right)$, such that the grip angle is never allowed to enter the "small angle" region relative to the toggle position. Using these values to inform the valid range of the rigid toe angle, we choose $\Psi=11 \pi / 12 \mathrm{rad}$, which is the midpoint of the valid range. The nominal base length is computed as $\tilde{b}=3.464$ using Eq. (6). We choose a nominal link length as $\tilde{L}=\tilde{b} / 2$, so that the mechanism is approximately square when hanging below the rotorcraft. Finally, we choose a nominal value for the wind-disturbance center-ofpressure at $\tilde{a}=3.255$; this value is chosen because it is the actual value of our 
experimental case study used later. Fig. 6 shows the results of varying each design parameter one by one, starting from the nominal design, while holding the remaining parameters constant. As expected, we see an increase in disturbance rejection capability with an increase in $\tilde{L}$ and with a decrease in $\theta_{\min }$. Starting from the nominal design, we can get an increase in disturbance rejection by increasing $\tilde{b}$ and decreasing $\Psi$, with bigger potential gains by decreasing $\Psi$. We also see that each of these last two terms have lower and upper bounds in their allowable values, which are imposed by the other values in the nominal design.

To perform an optimization in which the design parameters can be varied simultaneously, we utilize the MATLAB optimization function fmincon to implement our governing equations, and use normalized force disturbance as our metric to be maximized. With our understanding that the optimization routine will always drive $\theta_{\text {min }}$ to zero, and will always drive $\tilde{L}$ forever upward, we remove those quantities from the optimization and simply set them at constant values of $\tilde{L}=\tilde{b} / 2$ and $\theta_{\min }=\pi / 18 \mathrm{rad}$. In the optimization, we use many randomly chosen initial conditions, which are first checked for validity, and then allow the numerical convergence to occur. Convergence always occurs to the same optimal parameter set, regardless of the initial condition. We find that the optimal normalized base length is $\tilde{b}=2$, and that the optimal rigid toe angle is described by the function:

$$
\Psi_{\text {optimal }}=\frac{\pi}{2}+\theta_{\min }
$$

The optimal design is depicted in Fig. 5(b). In the optimal design, the mechanism's base length is equal to the diameter of the perch, resulting in the toes 

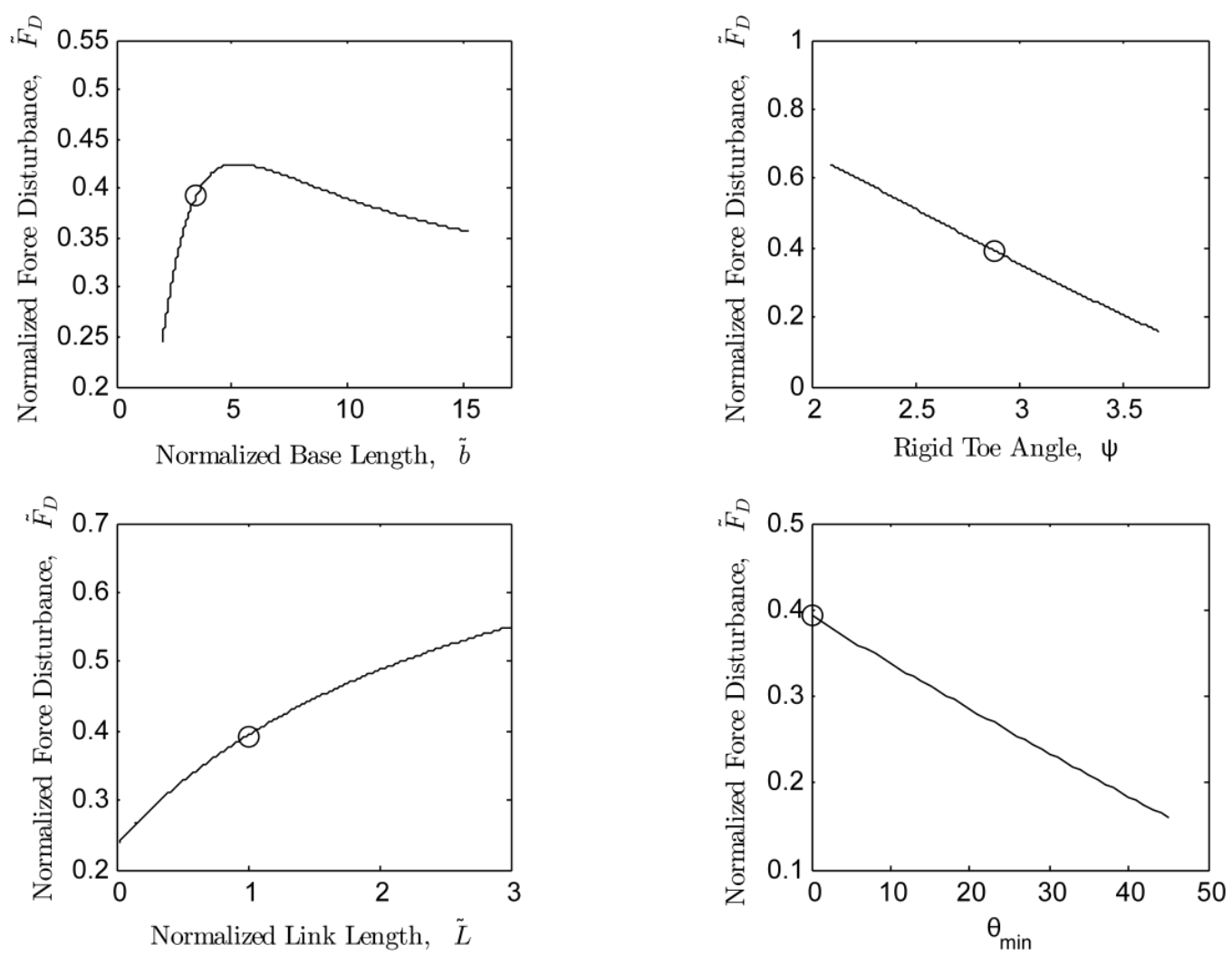

Fig. 6. Varying design parameters in nominal design for a single perch size. Effect of varying individual parameters on the normalized force disturbance $\widetilde{F}_{D}$, starting from the initial nominal design of (a). The nominal design is indicated with a circle. The parameters varied include: the base length $\widetilde{b}$, the rigid toe angle $\psi$, the link length $\widetilde{L}$, and the minimum grip angle $\theta_{\min }$ (in degrees). Each parameter is varied individually while holding all other parameters at the nominal values $\left(\tilde{b}=3.464, \tilde{L}=1.732, \theta_{\text {min }}=0\right.$, and $\tilde{a}=3.255$ ).

gripping the perch on the sides, and the rigid toe angle is such that the toes contact the perch at the instant when the mechanism has collapsed as close to the toggle position as is allowed by $\theta_{\min }$. Fig. 7 shows the results of varying each design parameter one by one, starting from the optimized design, while holding the remaining parameters constant. As part of the "optimized" design, we arbitrarily select $\tilde{L}=\tilde{b} / 2$ and $\tilde{a}=3.255$ as before. From the optimized design, we see that it is neither possible to increase nor decrease $\tilde{b}$ and still get a valid mechanism; decreasing $\tilde{b}$ would prohibit the toes from enclosing the 

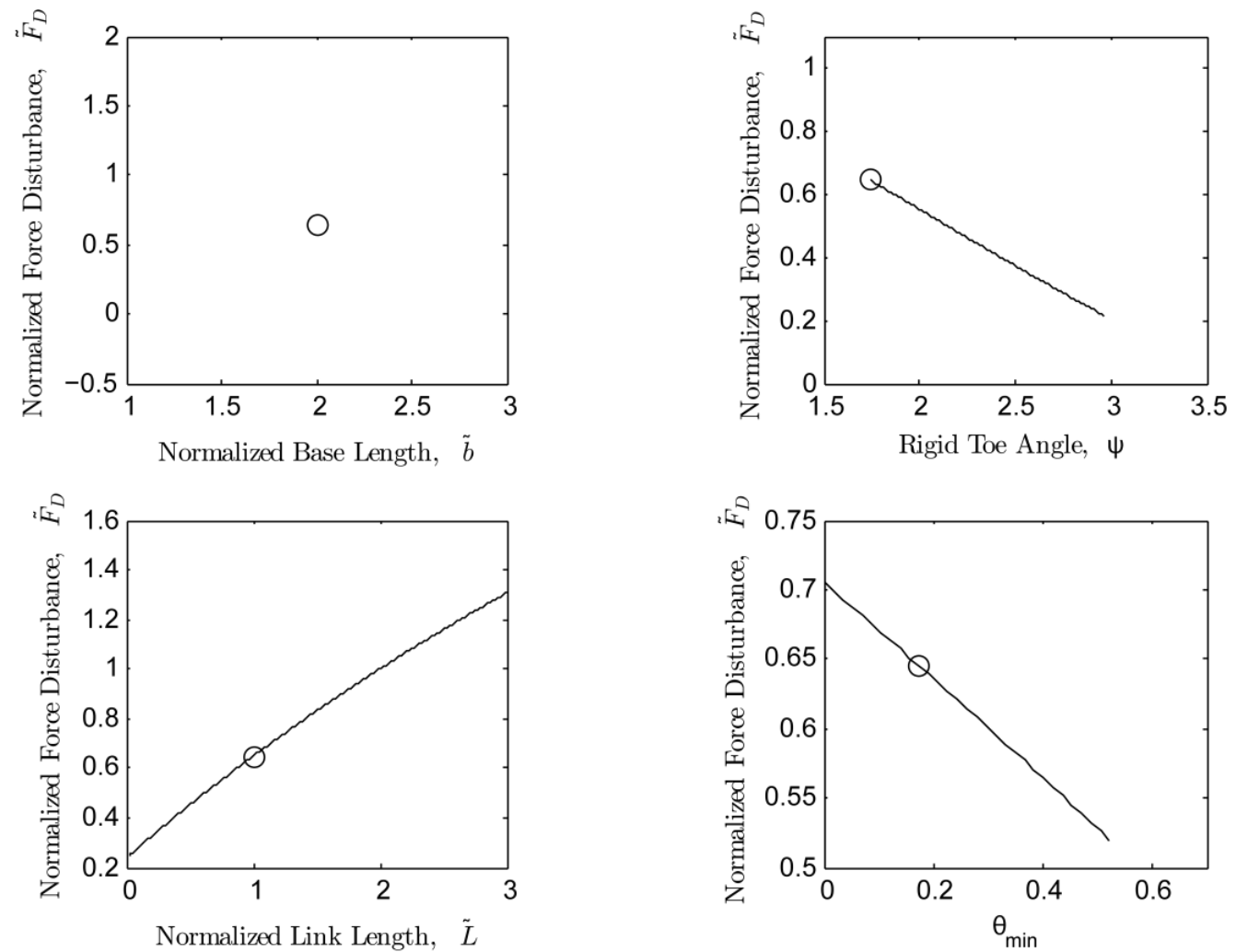

Fig. 7. Varying design parameters in optimal design for a single perch size. Effect of varying individual parameters on the normalized force disturbance $\widetilde{F}_{D}$, starting from the optimized design of Fig. 5(b). The parameters varied include: the base length $\tilde{b}$, the rigid toe angle $\psi$, the link length $\widetilde{L}$, and the minimum grip angle $\theta_{\min }$. The optimized design is indicated with a circle (the choices of $\tilde{L}$ and $\theta_{\min }$ are still user-defined and arbitrary). Each parameter is varied individually while holding all other parameters at the initial values $\left(\tilde{b}=2, \tilde{L}=1, \theta_{\min }=\frac{\pi}{18} \mathrm{rad}\right.$, and $\left.\tilde{a}=3.255\right)$.

perch, and increasing $\tilde{b}$ would result in the toes not making contact with the perch. It is possible to increase the rigid toe angle, but it would result in an inferior mechanism.

As expected, we would still realize improved disturbance rejection by increasing $\tilde{L}$ or decreasing $\theta_{\text {min }}$. Fig. 8 shows how the distance to the center of pressure $\tilde{a}$ affects the force disturbance that can be rejected. This parameter is a function of the aerodynamic properties of the rotorcraft, as well as the radius of the perch, and is thus not actually a 


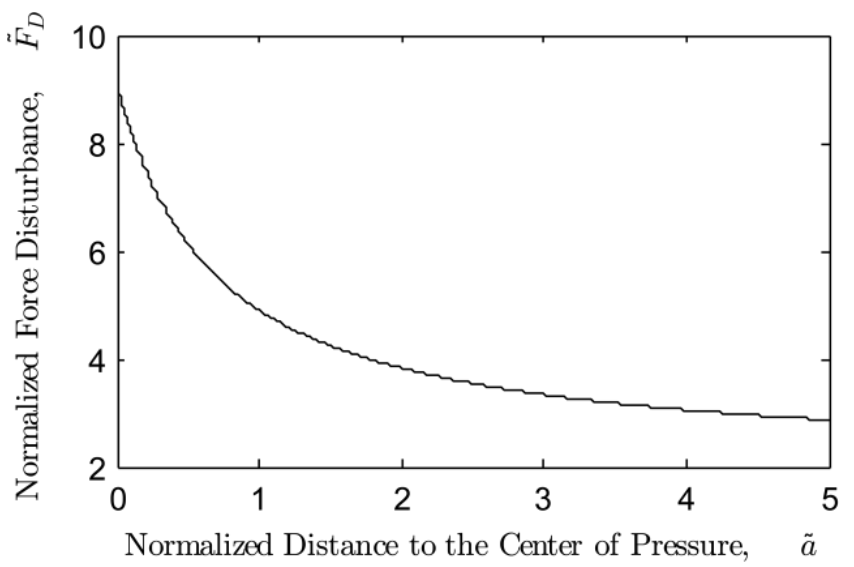

Fig. 8. Normalized force disturbance $\tilde{F}_{D}$ vs. distance to the center of pressure $\tilde{a}$ (with $\tilde{b}=2, \theta_{\min }=\frac{\pi}{18} \mathrm{rad}$, and $\tilde{L}=1$ ).

mechanism design parameter that can be optimized. However, we should understand the role of this parameter on our mechanism's ability to reject disturbances.

We observe that significant increases in disturbance rejection can be achieved if the distance between the center of pressure and the top of the perching mechanism can be minimized.

We summarize the optimal design process for a single perch size as follows:

1. Set the base length $b$ to be equal to the diameter of the perch (make it slightly larger for a small factor of safety).

2. Set the toe length $T$ to be equal to the radius of the perch (make it slightly larger for a small factor of safety).

3. Choose the minimum grip angle $\theta_{\min }$ to be as small as allowable to avoid toggle positions, ideally based on strength and tolerance analysis of the resulting mechanism. 
4. Set the rigid toe angle $\Psi$ to be $\pi / 2 \operatorname{rad}\left(90^{\circ}\right)$ larger than the minimum grip angle $\theta_{\min }$

5. Choose the link length $L$ to be as large as possible, given payload constraints.

6. Mount the perching mechanism on the rotorcraft such that the kinematic "top" of the mechanism is as high as possible in order to minimize its distance, $a$, to the center of pressure of the rotorcraft.

\section{Optimizing for a Range of Perch Sizes}

Rather than designing a mechanism that is optimized to perch on a single perch, it is probably more desirable to be able to perch on an entire inclusive range of perch sizes. To optimize for a range of perch sizes, we first define the range of parameters for which the mechanism/rotorcraft system will achieve a valid grasp (as depicted in Fig. 3) throughout the entire range. We then use our knowledge gained during the optimization for a single perch to inform our optimization for a range of perch sizes. We continue to use the normalized force disturbance $\tilde{F}_{D}$ that can be rejected as our design metric that is to be maximized.

To consider a range of perch sizes, we will normalize the radius of a given perch in the range relative to the radius of the largest perch in the range, such that every perch in the range is described by a dimensionless scaling parameter $s \in(0,1]$. Thus, the largest perch in the range is always represented by $s=1$, and all smaller perches are represented by some $0<s<1$.

We learned two important facts in the previous section about optimizing the mechanism for a given perch. First, we learned that the base $b$ should be made as small as possible, which also makes the effective toe length as small as possible. For a range of 
perch sizes, the smallest base length that will result in valid perches throughout the entire range is achieved by choosing the base equal to the diameter of the largest perch in the range: $\tilde{b}=2$.

Second, we learned that it is always desirable to minimize the grip angle $\theta$, both to increase the mechanical advantage of the mechanism, and to reduce the moment arm of any force disturbances due to wind. Avoiding the mechanism's toggle positions throughout an entire range of perch sizes is achieved by choosing the grip angle to be equal to the minimum grip angle $\theta_{\min }$ for the smallest perch in the range. The result is that the grip angle on all other larger perches in the range will be larger than the minimum grip angle, due to the need of the mechanism to expand to accommodate larger perches. Recall that $\theta_{\min }$ is set by the designer to avoid the mechanism's toggle positions. A consequence of breaching toggle positions includes losing the free movement of mechanism needed for both ascent and descent. Thus, we must also ensure that the largest perch in a given range does not cause the mechanism to breach the fully open toggle position, which we will also choose to avoid by the same $\theta_{\min }$ (although a different value could be used, considering the different loading conditions on the mechanism in the two toggle positions). Avoiding these two toggle positions, taken together, restricts the range of perch sizes that can be gripped by a given mechanism. Since the grip angle for the largest perch in the range is always $\beta=0$, the grip angle for the smallest perch, $\beta_{\text {small }}$, is equal to the total $\theta$ swept out from the largest to the smallest perch, which in turn is limited by avoiding the upper and lower toggle positions. Thus, the smallest perch that can be gripped for a given designer-specified $\theta_{\min }$ is calculated as: 


$$
s_{\text {min }}=\tan \left(\frac{\max \beta_{\text {small }}}{2}\right) \frac{\tilde{b}}{2}=\tan \left(\frac{\frac{\pi}{2}-2 \theta_{\min }}{2}\right) \frac{\tilde{b}}{2}
$$

Fig. 9 shows how setting a more conservative (i.e., larger) minimum grip angle restricts the range of perch sizes that can be gripped.

Fig. 10 shows the nonlinear relationship between the maximum normalized force disturbance, $\tilde{F}_{D}$, that can be rejected through an inclusive range of perch sizes, for four different ranges. A range of perch sizes is denoted by $s=\left[s_{\text {small }} S_{\text {large }}\right]$. We observe that increasing the size of the range results in a decrease in the force disturbance that can be rejected on the largest perch in the range, with a nearly symmetric corresponding increase in the disturbance that can be rejected on the smallest perch in the range. We also observe that the median disturbance that can be rejected, which occurs at approximately the median perch size within a given range of perch sizes, is quite insensitive to the size of the range. Although the normalized force disturbance values reported in the figure are a function of three somewhat-arbitrary parameters $\tilde{L}, \theta_{\min }$, and $\tilde{a}$, the overall trends remain the same for different values.

In Fig. 11, we consider the effect of varying the normalized link length $\tilde{L}$ on the maximum normalized force disturbance $\tilde{F}_{D}$, using the same ranges of perch sizes shown in Fig. 10. Note the values reported at $\tilde{L}=1$ are the same as those reported in Fig. 10. As expected, we observe that increasing the link length increases the force disturbance that can be rejected across designs. However, we also observe that the increases in disturbance rejection are greater at the small end of the perch-size range with increased sensitivity to link length as the range of perch sizes increases, and less pronounced at the large end of the perch-size range with decreased sensitivity to link length as the range of 


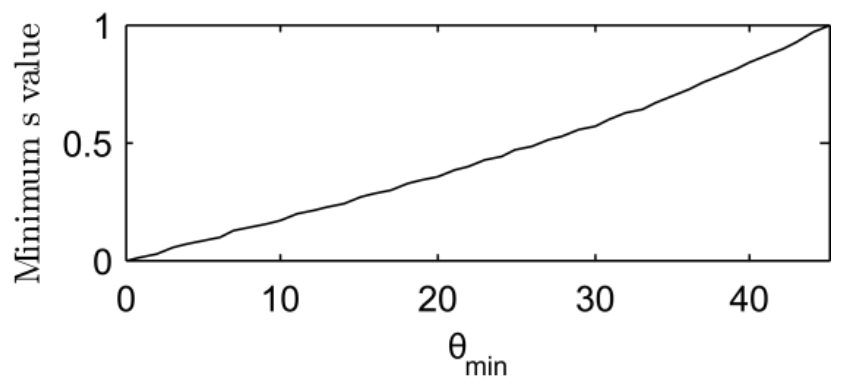

Fig. 9. Minimum relative perch size $s$ vs. minimum grip angle $\theta_{\min }$ (with $\tilde{b}=2$ ). By increasing the minimum grip angle, the range of achievable perch sizes is decreased.

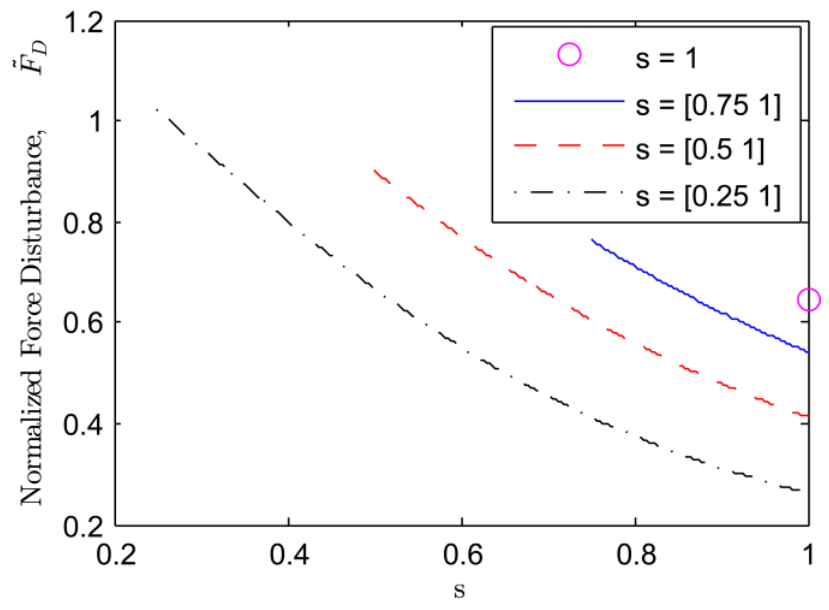

Fig. 10. Normalized force disturbance vs. relative perch size for four different mechanisms that have each been optimized for a specified range of perch sizes (with $\tilde{L}=1, \theta_{\min }=\frac{\pi}{18} \mathrm{rad}$, and $\tilde{a}=3.255$ in all cases). The "range" $s=1$ corresponds to the optimal design for a single perch size. 


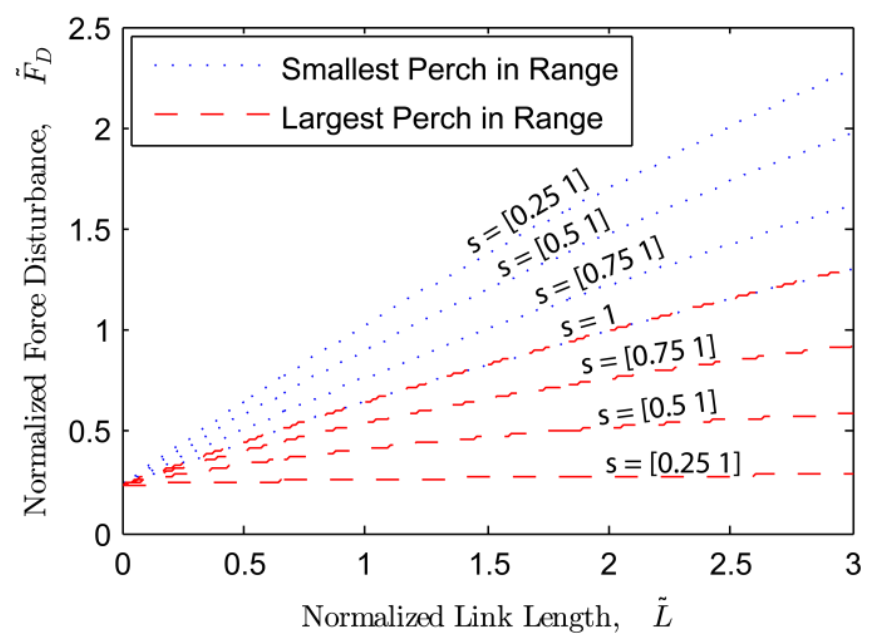

Fig. 11. Normalized force disturbance vs. normalized link length for four different mechanisms that have each been optimized for a specified range of perch sizes (with $\theta_{\min }=\frac{\pi}{18} \operatorname{rad}$ and $\tilde{a}=3.255$ in all cases). The "range" $s=1$ corresponds to the optimal design for a single perch size.

perch sizes increases. We observe that the difference in disturbance-rejection capability between the smallest and largest achievable perches within a given optimized range increases with increasing link length. This seems to confirm the significant effect in lowering the effective moment arm of the force disturbance about the perch by decreasing the smallest perch size, which allows the mechanism to settle deeper into the perch. This also decreases the force disturbance by the largest perch due to the increase in moment arm to allow smallest perch sizes to not reach the toggle position.

In Fig. 12, we consider the effect of varying the minimum grip angle $\theta_{\min }$ on the maximum normalized force disturbance $\tilde{F}_{D}$, using the same ranges of perch sizes shown in Fig. 10. Note the values reported at $\theta_{\min }=10^{\circ}$ are the same as those reported in Fig. 10. We see that the smaller perch's force disturbance always exceeds that of the larger perch as expected. We also see that decreasing the $\theta_{\min }$ that we will allow always results in an increase in disturbance rejection capability, as expected. 


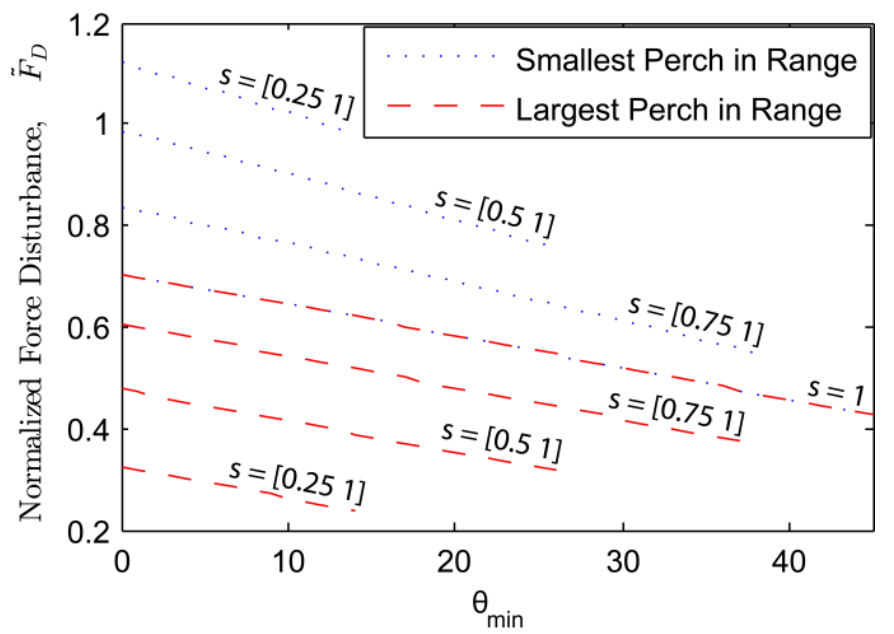

Fig. 12. Normalized force disturbance vs. minimum grip angle (in degrees) for four different mechanisms that have each been optimized for a specified range of perch sizes (with $\tilde{L}=1$ and $\tilde{a}=3.255$ in all cases). The "range" $s=1$ corresponds to the optimal design for a single perch size.

We summarize the optimal design process for a range of perch sizes as follows:

1. Set the base length $b$ equal to the diameter of the largest perch in the range (make it slightly larger for a small factor of safety).

2. Set the toe length $T$ to be equal to the radius of the largest perch in the range (make it slightly larger for a small factor of safety).

3. Choose the minimum grip angle $\theta_{\min }$ to be as small as allowable to avoid toggle positions, ideally based on strength and tolerance analysis of the resulting mechanism.

4. Set the grip angle $\theta$ to be equal to $\theta_{\min }$ on the smallest perch in the range.

5. Set the rigid toe angle $\Psi$ based on the $b$ value and the $\theta$ value for the smallest perch, using Eqs. (6) and (7). 
6. Choose the link length $L$ to be as large as possible, given payload constraints.

7. Mount the perching mechanism on the rotorcraft such that the kinematic top plate of the mechanism is as high as possible in order to minimize its distance to the center of pressure of the rotorcraft. 


\section{CHAPTER 5}

\section{PROTOTYPE AND EXPERIMENTAL VERIFICATION}

Using a Sky Crawler RC helicopter (Excalibur, Yonkers, NY), we utilized the procedures for finding the optimal perching mechanism for a range of perch sizes to create a prototype. First, we gathered drag and lift force data from a wind tunnel to model the forces to which the helicopter/mechanism system will be subjected. Then, we experimentally estimate the vertical location of the center of pressure, $a$. Link lengths are constrained using the published payload limits of the Sky Crawler. Using the values found for this specific helicopter, we designed and constructed the optimal mechanism for a range of perch sizes.

To understand the drag and lift forces that would be exerted onto the helicopter, we utilized the undergraduate wind tunnel at the University of Utah (Fig. 13). In the wind tunnel, the helicopter was mounted on top of a force sensor that measures drag and lift forces and air pressure. Knowledge of the drag allows us to understand the effective force disturbance acting on the helicopter for a given wind speed. The lift due to air flow causes the force of the helicopter exerted onto the mechanism to deviate from its weight $W$. The results of the wind-tunnel experiment for drag and lift are shown in Fig. 14 and Fig. 15, respectively.

To find the distance to the center of pressure, $a$, we used the assumption that the center of pressure is approximately at the same vertical position as the center of mass 


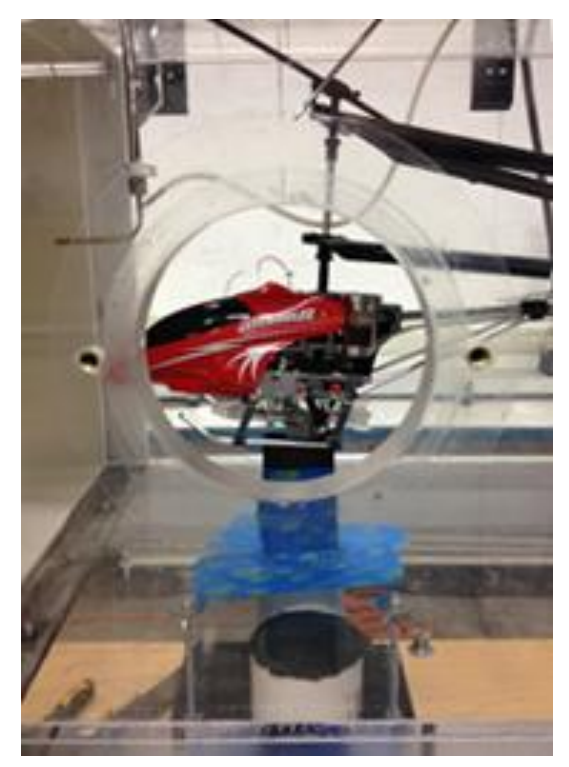

Fig. 13. Helicopter in wind tunnel. A wind tunnel was used to quantify the drag and lift forces exerted on the RC helicopter at specified wind speeds.

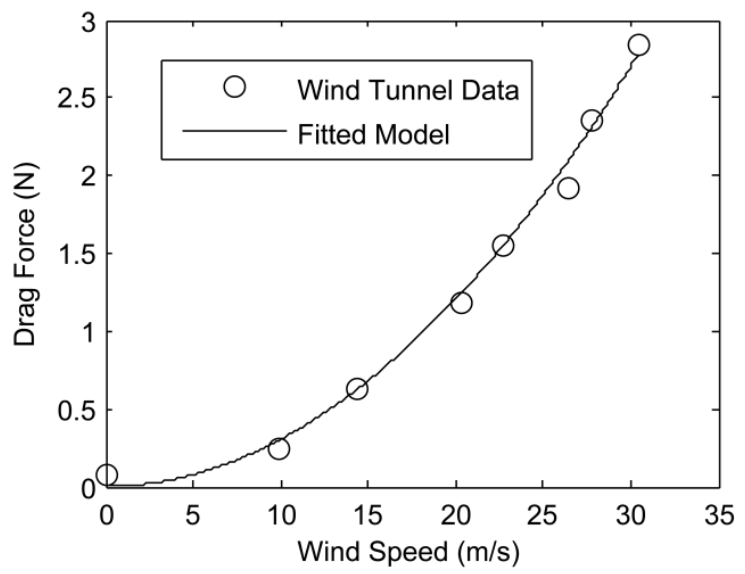

Fig. 14. Drag force vs. wind speed obtained in a wind tunnel. Drag is modeled as a quadratic function of wind speed: $F_{D}=C_{D} v^{2}$. The coefficient $C_{D}=3.00 \times 10^{-3} \mathrm{~N} \cdot \mathrm{s} / \mathrm{m}$ was calculated using polynomial fitting. The quadratic model is a good fit to the experimental data. 


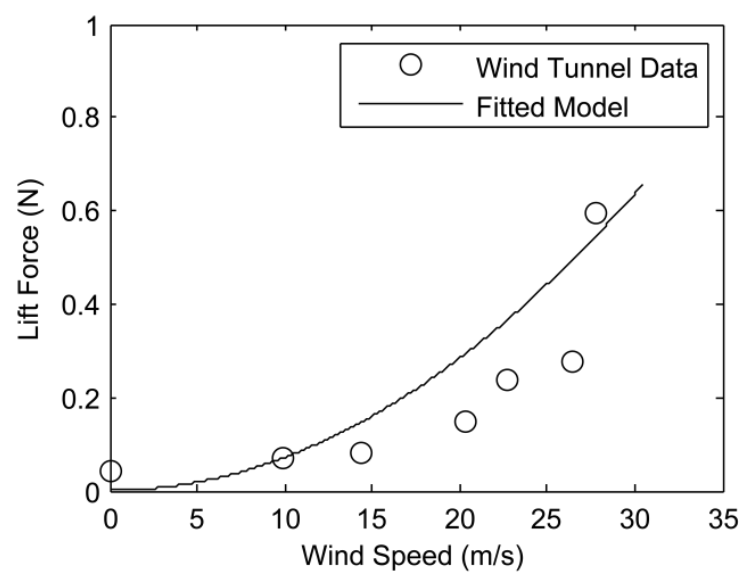

Fig. 15. Lift force vs. wind speed obtained in a wind tunnel. Lift is modeled as a quadratic function of wind speed: $F_{L}=C_{L} v^{2}$. The coefficient $C_{L}=7.05 \times 10^{-4} \mathrm{~N} \cdot \mathrm{s} / \mathrm{m}$ was calculated using polynomial fitting. The quadratic model can be used as a coarse approximation, but it over-predicts the data in many cases.

(otherwise large pitching moments would result during forward flight). This allowed us to do a simple plumb-bob experiment in which the helicopter is held from a string from a single point and a vertical line is drawn downward from that point, with the understanding that the center of mass is somewhere on that line (Fig. 16). This is repeated by attaching the string to another point, generating another vertical line. The center of mass is then located at the intersection of the two lines. The distance from this intersection point to the base of the helicopter is thus the location of the center of mass, which we can use as an approximation of the value for $a$. We estimate a value of $a=$ $62 \mathrm{~mm}$.

We designed our mechanism for use on a range of perches in which the largest perch has a diameter of $42 \mathrm{~mm}(r=21 \mathrm{~mm})$, which corresponds to a dimensionless perch size of $s=1$ by definition, and the smallest perch has a diameter of $21 \mathrm{~mm}$ $(r=10.5 \mathrm{~mm})$, which corresponds to a dimensionless perch size $s=0.5$. The perches 


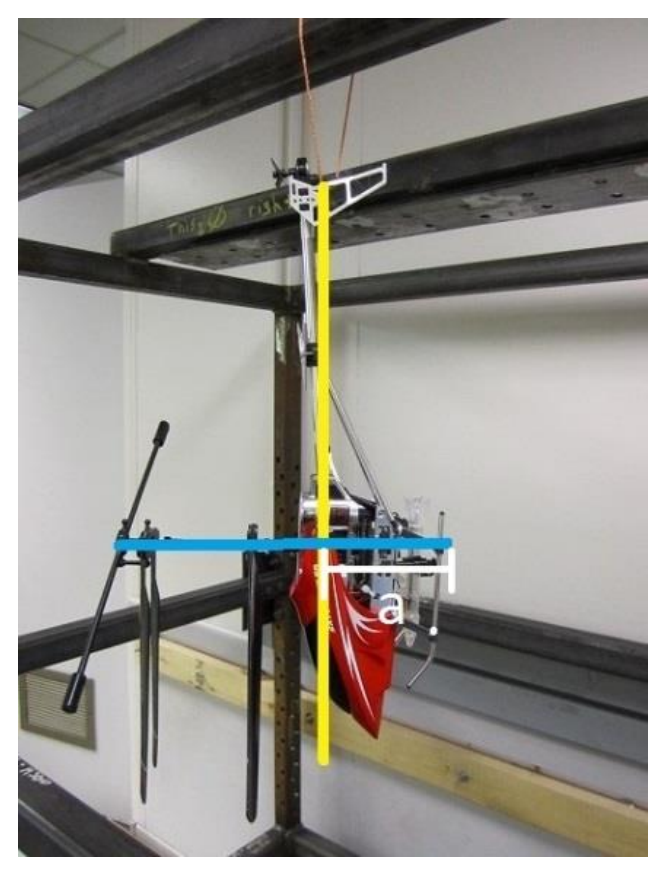

Fig. 16. Center of mass of the RC helicopter. The center of mass of the helicopter is found by hanging the helicopter from two different points, with an understanding that the center of mass will lie somewhere on the vertical line below the given hanging point. The center of pressure is then approximated at the center of mass, at $a=62 \mathrm{~mm}$.

we use here are made of PVC pipe, which is available in a variety of diameters.

We chose our $\theta_{\min }$ iteratively through a series of prototypes and pilot tests. We settled on a $\theta_{\min }=0.262 \mathrm{rad}\left(15^{\circ}\right)$ because we found that if we allowed the mechanism to collapse beyond that value, the compliance in the links and joints would sometimes allow the mechanism to violate the fully collapsed toggle position. This same $\theta_{\min }$ was more than sufficient to prohibit the mechanism from violating the fully open toggle position, since the weight of the hanging perching mechanism is much less than the weight of the helicopter. We designed a hard mechanical stop to prohibit the mechanism from violating the fully open toggle position when hanging below the helicopter. The fully collapsed toggle position is avoided naturally through design, provided we do not attempt to perch on perches outside of (i.e., smaller than) the range for which the 
mechanism was designed.

The complete system prototype was created using two perching mechanisms (one mechanism per "foot") with each possessing three linkages and three toes, and these two mechanisms are joined to a common platform. To rigidly connect the platform to the helicopter, snap clips were created on the top of the platform to enable the platform to snap on to the helicopter's skids. We used the method of Section IV-B to choose a base length of $b=42 \mathrm{~mm}$, a toe length of $T=28 \mathrm{~mm}$ (which has a $5 \%$ factor of safety), and a rigid toe angle of $\Psi=2.48 \mathrm{rad}\left(142^{\circ}\right)$. For the link length of our mechanism, we arbitrarily chose the value $L=25 \mathrm{~mm}$, and we verified that this value was less than the maximum value such that the total prototype weight (including the two feet and the connecting/mounting platform) was still within the helicopter's payload (the maximum allowable value based on payload is $L=45 \mathrm{~mm}$ ).

SolidWorks renderings of the final mechanism (i.e., a single foot), perching on the large and small perches, are shown in Fig. 17. The prototype was created in ABS plastic using a 3D printer. Each joint was reamed using a $1.5-\mathrm{mm}$ high-speed steel chucking reamer to place a low-friction pin joint that ensures free movement throughout the mechanism's ascent and descent onto a perch. Joints were also sanded down to minimize friction created from imperfections in the 3D printing. Table 1 shows the parameters used in the final mechanism design and analysis.

In order to increase the friction between the mechanism and the perch, we coated the toes and bottom plate of the mechanism in Mold Max 40 (Smooth-On, Easton, PA), which is a condensation-cure silicone rubber compound. The compound was mixed to data-sheet specifications and coated on the toes and base plate with a brush and then 


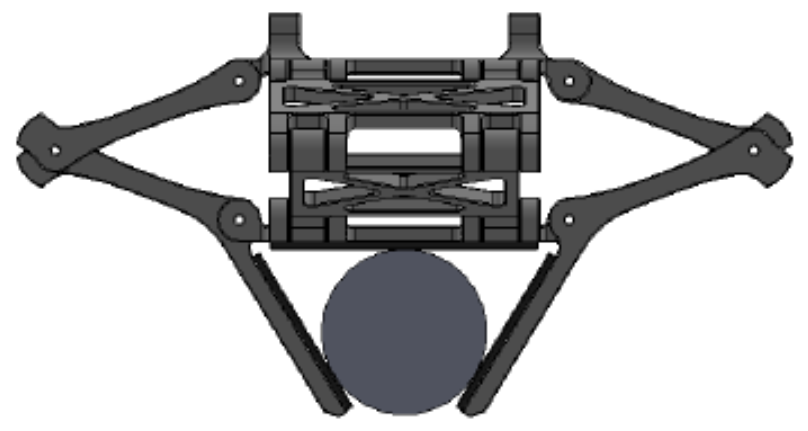

(a)

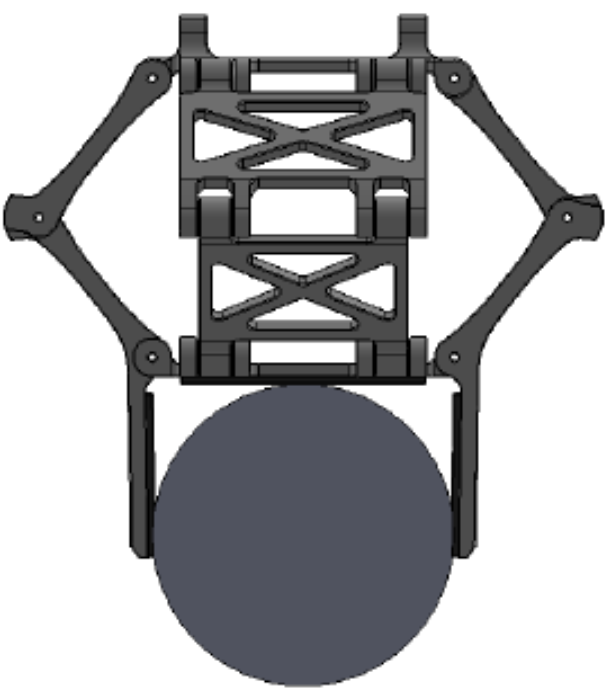

(b)

Fig. 17. Model of the prototype, optimized for the range of perch sizes of $s=[0.51]$. (a) shows the prototype on the smallest perch in range, and (b) shows the prototype on the largest perch in range.

Table 1. Parameters used for prototype design.

\begin{tabular}{|c|c|c|}
\hline Parameter & Value & Units \\
\hline Weight of Helicopter and Mechanism System, $W$ & 19.3 & $\mathrm{~N}$ \\
\hline Coefficient of Static Friction, $\mu$ & 0.35 & - \\
\hline Link Length, $L$ & 0.025 & $\mathrm{~m}$ \\
\hline Base, $b$ & 0.042 & $\mathrm{~m}$ \\
\hline Rigid Toe Angle, $\psi$ & 2.48 & $\mathrm{rad}$ \\
\hline Minimum Grip Angle, $\theta_{\min }$ & 0.262 & $\mathrm{rad}$ \\
\hline Distance to Center of Pressure, $a$ & 0.062 & $\mathrm{~m}$ \\
\hline
\end{tabular}


allowed to fully cure before first use.

The static coefficient of friction $\mu$ between the toes of the mechanism and the perch was found using a simple slip test between the material used to coat the toes and the material used for the perches. The static coefficient of friction was estimated by slowly inclining the two materials and measuring the angle $\varphi$ above horizontal at which a slip occurs. The static coefficient of friction is then calculated as $\mu=\tan (\varphi)$, which we measured by using the average of 10 trials to have a value of $\mu=0.35$.

To experimentally evaluate the disturbance-rejection capability of the perching mechanism, we conducted an experiment in which a string was attached to the back of the helicopter body at approximately the location of the center of pressure. The string left the helicopter body horizontally, and then was routed around a smooth PVC pipe acting as a pulley, such that precision weights could be applied at the end of the string, and the downward force due to their weight is converted into a rearward horizontal force on the helicopter. For each of the large and small perches, an experiment was conducted in which the helicopter was manually perched and then weight was slowly added to the string until slip in the grip was observed. The force value was recorded, and the process was repeated for a total of 10 trials per perch. For the largest perch, the disturbance force (mean \pm standard deviation) at which slip occurred was $327 \pm 38 \mathrm{mN}$. This value of force corresponds to a wind speed of approximately $10 \mathrm{~m} / \mathrm{s}$ (neglecting lift effects). For the parameters used in the prototype, our analytical model predicts a maximum disturbance of $293 \mathrm{mN}$, which under-predicted our actual mean value by $10 \%$, but was close to the worst-case value we observed in the 10 trials. For the smallest perch, the disturbance force at which slip occurred was $583 \pm 42 \mathrm{mN}$. This value of force corresponds to a wind 
speed of approximately $15 \mathrm{~m} / \mathrm{s}$ (neglecting lift effects). Our analytical model predicts a maximum disturbance of $710 \mathrm{mN}$, which over-predicted our actual mean value by $22 \%$, but was close to the best-case value we observed in the 10 trials. These experiments suggest our analytical model is valid for the purposes of perching-mechanism design, but a factor of safety should be included.

In Fig. 18, we show our mechanism enabling our helicopter to make successful perches on both the largest and smallest perch sizes in the desired range. In the demonstration, the helicopter is hung from a string like a marionette and manually made to descend and ascend with minimal control.

In our analysis, we assumed that perching failure would be due to a wind disturbance coming from the front or back of the rotorcraft, thus causing the grip to slip and rotate. To confirm this assumption, we performed an additional experiment to test the ability to reject disturbances coming from the side, with the conjecture that much larger forces will be rejected from this direction. We repeated the hanging-weight experiment described above, but this time by attaching the string with the hanging weights to the side of the helicopter, but at approximately the same height relative to the perch. The results for 10 trials were that the large perch rejects $571 \pm 33 \mathrm{mN}$, and the small perch rejects $1068 \pm 64 \mathrm{mN}$. These values represent a $75 \%$ increase for the large perch and $83 \%$ increase for the small perch compared to the back-weighted experiment described previously. These values confirm our assumptions that the helicopter is most vulnerable to wind disturbances from the front and back of the rotorcraft as oppose to from the side. 


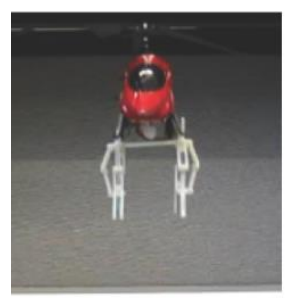

(a) $\mathrm{t}=0 \mathrm{sec}$

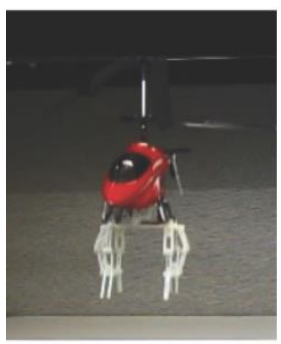

(a) $\mathrm{t}=0 \mathrm{sec}$

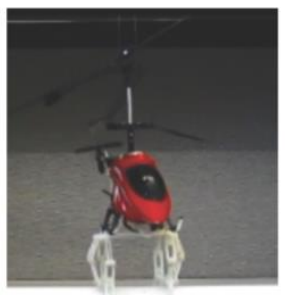

(b) $\mathrm{t}=1 \mathrm{sec}$

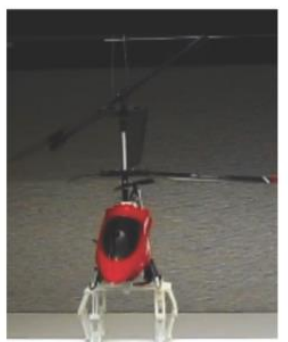

(b) $\mathrm{t}=0.6 \mathrm{sec}$

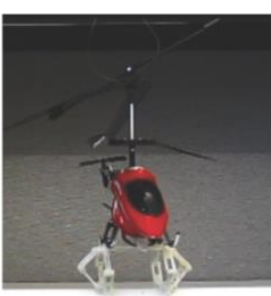

(c) $\mathrm{t}=2.2 \mathrm{sec}$

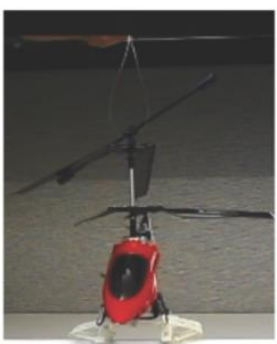

(c) $\mathrm{t}=1.9 \mathrm{sec}$
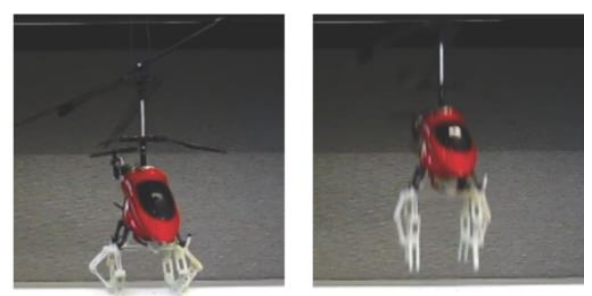

(d) $\mathrm{t}=3.4 \mathrm{sec}$

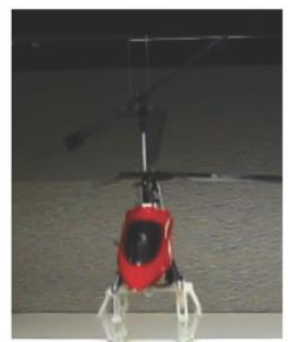

(d) $t=4.2 \mathrm{sec}$ (e) $\mathrm{t}=3.7 \mathrm{sec}$

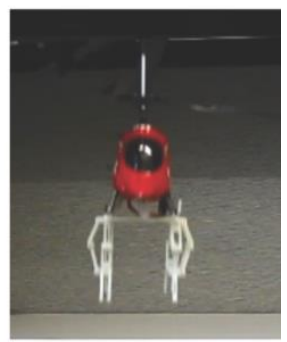

(e) $\mathrm{t}=4.5 \mathrm{sec}$

Fig. 18. Video images of the descent and ascent of helicopter with attached perching mechanism on $42 \mathrm{~mm}$ (top row) and $21 \mathrm{~mm}$ (bottom row) diameter PVC perches. The system is manually lowered onto the perch using a string in (a)-(b), the string is allowed to go completely slack in (c) to allow the complete weight of the helicopter to generate a grip and pause there, and then the string is used to lift the helicopter off the perch in (d)(e). 


\section{CHAPTER 6}

\section{CONCLUSION AND FUTURE WORK}

In this thesis, we proposed a simple mechanism based on a Sarrus linkage that enables a rotorcraft to perch on cylindrical perches using only the weight of the rotorcraft to maintain a grip on the perch. We provided an analysis of the mechanism's kinematics, we presented the static force equations that describe how the weight of the rotorcraft is converted into grip force onto the perch, and we described how grip forces relate to the ability to reject horizontal disturbances such as wind gusts. The mechanism was optimized for use on a single perch size, and then for a range of perch sizes. We concluded by constructing a prototype mechanism optimized for a range of perches in which the smallest perch in the range is half the diameter of the largest perch in the range. We demonstrated the usefulness of the mechanism with a remote-controlled helicopter through a series of experiments. For the helicopter, which has a weight of $19.1 \mathrm{~N}$, the mechanism was able to resist disturbances equivalent to wind speeds of $10 \mathrm{~m} / \mathrm{s}$ by design, and the mechanism is light enough to be lifted by the helicopter without utilizing its entire payload capacity.

In the future, it would be interesting to consider the same basic perching mechanism designed here, but without the assumption of straight rigid toes, which was made arbitrarily and for simplicity. It is possible that other toe geometries could lead to more desirable perching behavior. It would also be desirable to consider the use of 
different materials with higher strength-to-weight ratios, and to consider more optimized structural designs, again with the goal of reducing weight. Finally, adding highly compliant high-friction padding to the toes and palm of the mechanism could enable better gripping of perches with little impact on the weight budget. 


\section{REFERENCES}

[1] C. E. Doyle, J. J. Bird, T. A. Isom, J. C. Kallman, D. F. Bareiss, D. J. Dunlop, R. J. King, J. J. Abbott, and M. A. Minor, "An Avian-Inspired Passive Mechanism for Quadrotor Perching," IEEE/ASME Trans. Mechatronics, 18(2):506-517, 2013.

[2] C. E. Doyle, J. J. Bird, T. A. Isom, C. J. Johnson, J. C. Kallman, J. A. Simpson, R. J. King, J. J. Abbott, and M. A. Minor, "Avian-Inspired Passive Perching Mechanism for Robotic Rotorcraft," IEEE/RSJ Int. Conf. Intelligent Robots and Systems, pp. 4975-4980, 2011.

[3] Wikipedia. (2013). Sarrus Linkage, [Online]. Available: http://en.wikipedia.org/wiki/Sarrus_linkage.

[4] W. Danko, A. Kellas, and P. Y. Oh, "Robotic Rotorcraft and Perch-and-Stare: Sensing Landing Zones and Handling Obscurants," in IEEE Int. Conf. Adv. Robot., pp. 296-302, 2005.

[5] A. Hurst and E. Garcia, "Controller Design for a Morphing, Perching Aircraft," Proc. SPIE 7977, Active and Passive Smart Structures and Integrated Systems 2011, 79771L (May 02, 2011).

[6] J. Moore, R. Tedrake, "Control synthesis and verification for a perching UAV using LQR-Trees." IEEE/RSJ Int. Conf. Robotics and Automation, pp. 2235-2242, 2012.

[7] J. Moore, R. Tedrake, "Magnetic Localization for Perching UAVs on Powerlines," IEEE/RSJ Int. Conf. Intelligent Robots and Systems, pp.2700,-2707, 2011.

[8] A. L. Desbiens, A. T. Asbeck, and M. R. Cutkosky, "Landing, Perching and Taking Off From Vertical Surfaces," Int. J. Robot. Res., vol. 30, no. 3, pp. 355-370, 2011.

[9] A. L. Desbiens, A. T. Asbeck, and M. R. Cutkosky. "Scansorial Landing and Perching." Robotics Research. Springer Berlin Heidelberg, 169-184, 2011.

[10] D. Robertson and G. Reich. "Design and Perching Experiments of Bird-like Remote Controlled Planes." AIAA Structural Dynamics Conference. 2013.

[11] J. C. Goldin, 2011, "Perching Using a Quadrotor with Onboard Sensing," Master's Thesis, Department of Mechanical Engineering, Utah State University, Logan, Utah. 
[12] R. Cory and R. Tedrake, "Experiments in Fixed-wing UAV Perching," in Proc. AIAA Guid., Navigat., Control Conf., pp. 1-12., 2008.

[13] E. Glassman, A. L. Desbiens, M. Tobenkin, M. Cutkosky, R. Tedrake. "Region of Attraction Estimation for a Perching Aircraft: A Lyapunov Method Exploiting Barrier Certificates." IEEE Int. Conf. Robotics and Automation, pp. 2235 - 2242, 2012.

[14] M. L. Anderson, C. J. Perry, B. M. Hua, D. S. Olsen, J. R. Parcus, K. M. Pederson, and D. D. Jensen, "The Sticky-pad Plane and Other Innovative Concepts for Perching UAVs," in Proc. AIAA Aerospace Sci. Meeting, pp. 460-470, 2009.

[15] L. Daler, A. Klaptocz, A. Briod, M. Sitti, and D. Floreano, "A Perching Mechanism for Flying Robots Using a Fibre-Based Adhesive," IEEE Int. Conf. Robotics and Automation, pp. 4418-4423, 2013.

[16] A. Larson, 2009, "Development and Testing of an Active Perching System," Bachelor's Thesis, Department of Mechanical and Aerospace Engineering, Oklahoma State University, Stillwater, Oklahoma.

[17] R. J. Bachmann, F. J. Boria, R. Vaidyanathan, P. G. Ifju, and R. D. Quinn, "A Biologically Inspired Micro-vehicle Capable of Aerial and Terrestrial Locomotion," Mechanism Mach. Theory, vol. 44, pp. 513-526, 2009.

[18] M. Kovac, J. Germann, C. Hurzeler, R. Y. Siegwart, and D. Floreano, "A Perching Mechanism for Micro Aerial Vehicles," J. Micro-Nano Mechatronics, vol. 5, no. 4, pp. 77-91, pp. 460-470, 20102009.

[19] A. Klaptocz; L. Daler ; A. Briod; J.C. Zufferey.; D. Floreano, "An Active Uprighting Mechanism for Flying Robots," IEEE Trans. Robotics, vol.28, no.5, pp.1152,1157, 2012.

[20] E. S. Culler, G. C. Thomas, C. L. Lee, "A Perching Landing Gear for a Quadcopter," AIAA/ASME/ASCE/AHS/ASC Structures, Structural Dynamics and Materials Conference. 23-26 April 2012.

[21] J. R. Thomas, J.J. Polin, K. Sreenath, and V. Kumar, "Avian-Insprited Grasping for Quadrotor Micro UAVs," to appear IDETC/CIE, 2013.

[22] D. Mellinger, Q. Lindsey, M. Shomin, and V. Kumar, "Design, modeling, estimation and control for aerial grasping and manipulation," IEEE/RSJ Int. Conf. Intelligent Robots and Systems, pp. 2668-2673, 2011.

[23] P. E. Pounds, D. R. Bersak, and A. M. Dollar, "Stability of Small-scale UAV Helicopters and Quadrotors with added payload mass under PID Control," Autonomous Robots, vol. 33, no. 1-2, pp. 129-142, 2012. 
[24] V. Ghadiok, J. Goldin, and W. Ren, "On the design and development of attitude stabilization, vision-based navigation, and aerial gripping for a low-cost quadrotor," Autonomous Robots, vol. 33, no. 1-2, pp. 41-68, 2012.

[25] A. Hurst, A. Wickenheiser, and E. Garcia, "Localization and perching maneuver tracking for a morphing UAV," Position, Location and Navigation Symposium, pp. 1238-1245, 2008.

[26] J. C. Gomez and E. Garcia, "Morphing Unmanned Aerial Vehicles," Smart Mater. Struct., vol. 20, no. 10, 2008.

[27] A. Nagendran, W. Crowther, and R. Richardson, "Biologically Inspired Legs for UAV Perched Landings," Aerospace and Electronic Systems Magazine, vol. 27, no. 2, pp. 4-13, 2012. 